\title{
Suppression of macrophage infiltration into the conjunctiva by clodronate liposomes in experimental immune-mediated blepharoconjunctivitis
}

\author{
Atsuki Fukushima ${ }^{\mathrm{a}, *}$, Akemi Ozaki ${ }^{\mathrm{a}}$, Waka Ishida ${ }^{\mathrm{a}}$, Nico van Rooijen ${ }^{\mathrm{b}}$, \\ Kazuyo Fukata ${ }^{a}$, Hisayuki Ueno ${ }^{\mathrm{a}}$ \\ ${ }^{a}$ Laboratory of Immunology, Department of Ophthalmology, Kochi Medical School, Nankoku-city, 783-8505 Japan \\ ${ }^{\mathrm{b}}$ Department of Cell Biology and Immunology, Faculty of Medicine, Vrije Universiteit, Amsterdam, The Netherlands
}

Received 10 July 2004; revised 1 November 2004; accepted 7 December 2004

\begin{abstract}
Macrophages infiltrate the conjunctiva in severe cases of allergic conjunctivitis (AC) such as atopic keratoconjunctivitis (AKC). We established experimental immune-mediated blepharoconjunctivitis (EC) in Brown Norway (BN) rats as a model for severe types of AC. We investigated whether macrophage infiltration in the conjunctiva in this EC model is inhibited by clodronate liposomes ( $\mathrm{CL}_{2} \mathrm{MDP}$-lip). The numbers of ED1-positive but not ED2-positive macrophages in the conjunctivas were increased by the induction of EC. Subconjunctival injection of $\mathrm{CL}_{2} \mathrm{MDP}$-lip decreased the number of ED2-positive but not ED1-positive macrophages in the conjunctivas of naive rats. $\mathrm{CL}_{2} \mathrm{MDP}$-lip did not affect macrophages in the spleen. Subconjunctival injection of $\mathrm{CL}_{2} \mathrm{MDP}$-lip into EC-developing BN rats decreased the number of ED2-positive macrophages at all the time points. ED1-positive cell infiltration was inhibited when treatment was administered just prior to OVA challenge. Intravenous injection of $\mathrm{CL}_{2} \mathrm{MDP}_{\mathrm{O}}$-lip decreased the number of ED2-positive cells in the conjunctiva. Thus, we conclude that $\mathrm{CL}_{2} \mathrm{MDP}$-lip inhibits infiltration of macrophages into the conjunctiva within $24 \mathrm{~h}$ of antigen challenge.
\end{abstract}

(C) 2005 International Federation for Cell Biology. Published by Elsevier Ltd. All rights reserved.

Keywords: Clodronate liposomes; ED1; ED2; Experimental immune-mediated blepharoconjunctivitis; Macrophage

\section{Introduction}

Macrophages infiltrate the conjunctiva of various acute and chronic conjunctival diseases, including ocular cicatricial pemphigoid (OCP) (Foster and Sainz De La Maza, 2004). Increased expression of macrophage migration inhibitory factor (MIF), which induces accumulation of macrophages in the tissue, was demonstrated in the conjunctiva of OCP (Razzaque et al.,

\footnotetext{
* Corresponding author. Tel.: +8188 880 2391; fax: +8188 880 2392.

E-mail address: fukusima@med.kochi-u.ac.jp (A. Fukushima).
}

2004). In addition, CD68 positive macrophages produce chemokines in the conjunctiva of patients with active vernal keratoconjunctivitis (VKC) (El-Asrar et al., 2002). Thus, macrophages could play a role in maintaining the progression of conjunctival disease processes.

In allergic conjunctivitis (AC), eosinophils and mast cells (Church and McGill, 2002) are major effector cells. Macrophages have also been identified in the conjunctiva in severe kinds of $\mathrm{AC}$, such as atopic keratoconjunctivitis (AKC) (Foster et al., 1991). Infiltrating macrophages reportedly produce growth factors (Leonardi et al., 2000), and are therefore probably involved in the formation of giant papillae. However, the function of conjunctival macrophages in AKC patients is still unclear. 
We established experimental immune-mediated blepharoconjunctivitis (EC) in Brown Norway (BN) rats as a model for severe types of AC (Iwamoto et al., 2000; Yoshida et al., 1999). Eosinophils, macrophages and T cells infiltrate the conjunctiva in this EC model (Fukushima et al., 2003). Macrophages and T cells are abundant in the conjunctivas of patients with AKC (Foster et al., 1991), and the conjunctival cytokine profile (IFN- $\gamma>$ IL-4) in the EC model (Fukushima et al., 2003) is quite similar to that in AKC patients (Broekhuyse et al., 1997). Thus, this EC model appears to be an appropriate model for AKC (Fukushima et al., 2003).

Clodronate liposomes $\left(\mathrm{CL}_{2} \mathrm{MDP}\right.$-lip) have recently been shown to deplete macrophages in macrophageimplicated experimental disease models (Metz et al., 1997; Polfliet et al., 2002; Sakurai et al., 2003). Here, we investigated the inhibitory effects of $\mathrm{CL}_{2} \mathrm{MDP}$-lip on infiltration of macrophages into the conjunctiva in EC, to elucidate the role of macrophages in the initiation and maintenance of conjunctival inflammation.

\section{Materials and methods}

\subsection{Animals}

Male 8-12-week-old BN rats (Clea Japan, Tokyo, Japan) were maintained in a pathogen-free animal facility of the Kochi Medical School. All animal procedures conformed to the ARVO Resolution on Use of Animals in Research.

\subsection{Preparation of $C L_{2} M D P$-lip}

The method for preparation of $\mathrm{Cl}_{2} \mathrm{MDP}$ is published elsewhere (Van Rooijen and Sanders, 1994). $\mathrm{Cl}_{2} \mathrm{MDP}$ was a gift from Roche Diagnostics $\mathrm{GmbH}$, Mannheim, Germany. Phosphatidylcholine (LIPOID E PC) was obtained from Lipoid GmbH, Ludwigshafen, Germany. Cholesterol was purchased from Sigma Chemical Co. (USA). $\mathrm{CL}_{2}$ MDP-lip was prepared by dissolving 1,2 dioleolyl-sn-glycero-3-phosphocholine and cholesterol in a mixture of methanol and chloroform (1:1). After low-vacuum rotary evaporation at $37{ }^{\circ} \mathrm{C}$ to remove the organic phase, the lipids were mixed with clodronic acid $(1.9 \mathrm{~g})$ dissolved in phosphate-buffered saline $(0.6 \mathrm{M})$. The lipids sat at room temperature for $2 \mathrm{~h}$ in a nitrogenpurged chamber to induce liposome swelling. Liposomes were resuspended with water by sonication at room temperature for $3 \mathrm{~min}$. The resultant liposomes were washed at $10,000 \times g$ to $100,000 \times g$ in an ultracentrifuge twice for $30 \mathrm{~min}$ at $16{ }^{\circ} \mathrm{C}$. The milky liposomes were removed gently with a pipette, resuspended in $4 \mathrm{ml}$ of sterilized PBS, and stored in a nitrogen chamber for up to 1 week. Lipids were mixed with sterilized PBS to generate the PBS-liposomes.

\subsection{Antibodies for immunohistochemistry}

Anti-rat RT1B (OX-6) Ab was purchased from BD Pharmingen, San Diego, CA, USA. The target cells for OX-6 are MHC class II positive cells. Mouse anti-rat CD68 (ED1) (Dijkstra et al., 1985; van Rees et al., 1985) was purchased from Serotec, Kidlington, Oxford, UK. ED2 (Dijkstra et al., 1985; van Rees et al., 1985), which reacts with a membrane antigen $(175,160$, and $95 \mathrm{kDa})$ on resident rat macrophages, was purchased from BMA Biomedicals AG, Augst, Switzerland. Anti-major basic protein (MBP) was obtained from Biodesign Int., Saco, ME, USA. MBP, which is released from eosinophils, is a molecule which induces tissue damage. Biotinylated rabbit anti-mouse immunoglobulins (RAMG) were obtained from Dako, Copenhagen, Denmark.

\subsection{Establishment of OVA-specific T-cell lines}

The detailed method is described elsewhere (Fukushima et al., 2003), but briefly, ovalbumin (OVA grade V, Seikagaku Co., Tokyo, Japan) primed lymph node cells from BN rats immunized with OVA in complete Freund's adjuvant were stimulated repeatedly in vitro with OVA. Three days after the second stimulation, living cells were harvested by gradient centrifugation (Lymphocyte Separation Medium, Organon Teknika Corporation, West Chester, PA) and used for transfer. Flow cytometric analysis confirmed that the cells were $\mathrm{CD}^{+}, \mathrm{CD}^{+}$and CD8 ${ }^{-}$(data not shown).

\subsection{Induction and evaluation of $E C$}

EC was induced by adoptive transfer of $\mathrm{T}$ cell line cells (Fukushima et al., 2003). In brief, OVA-specific T cells $\left(5 \times 10^{6} /\right.$ rat $)$ were injected intravenously into syngeneic recipient rats. Four days later, they were challenged with OVA. The rats were sacrificed $24 \mathrm{~h}$ after OVA challenge and their eyes including conjunctivas were harvested for immunohistochemistry.

\subsection{Treatment protocol with $\mathrm{CL}_{2} \mathrm{MDP}$-lip}

$\mathrm{CL}_{2}$ MDP-lip or PBS as a control was injected either subconjunctivally or intravenously. Naive rats were injected with $20 \mu$ of $\mathrm{CL}_{2}$ MDP-lip or PBS subconjunctivally; 24,48 and $72 \mathrm{~h}$ after injection, conjunctivas were harvested for immunohistochemistry ( $n=6$ per group). EC-developing rats were injected subconjunctivally 24 and $48 \mathrm{~h}$ before OVA challenge or just prior to OVA challenge with $20 \mu \mathrm{l}$ of $\mathrm{CL}_{2}$ MDP-lip or PBS $(n=12$ per group). Intravenous injection of $\mathrm{CL}_{2} \mathrm{MDP}$-lip or PBS 
A

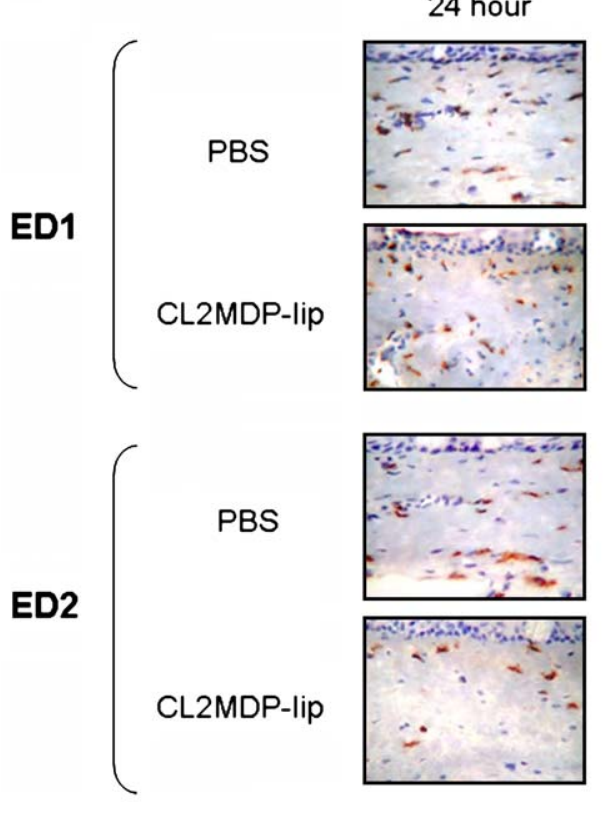

48 hour
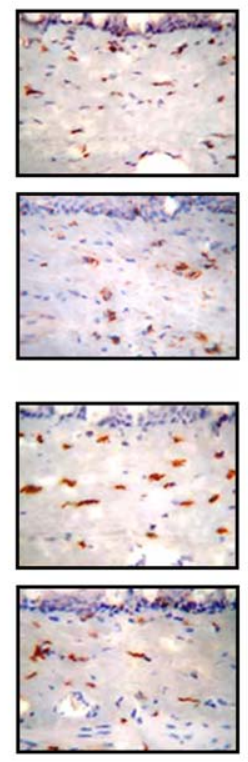

72 hour
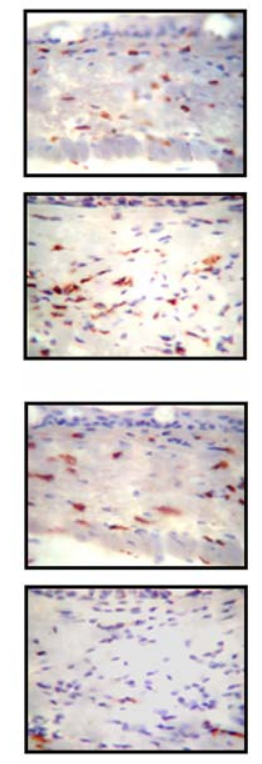

B
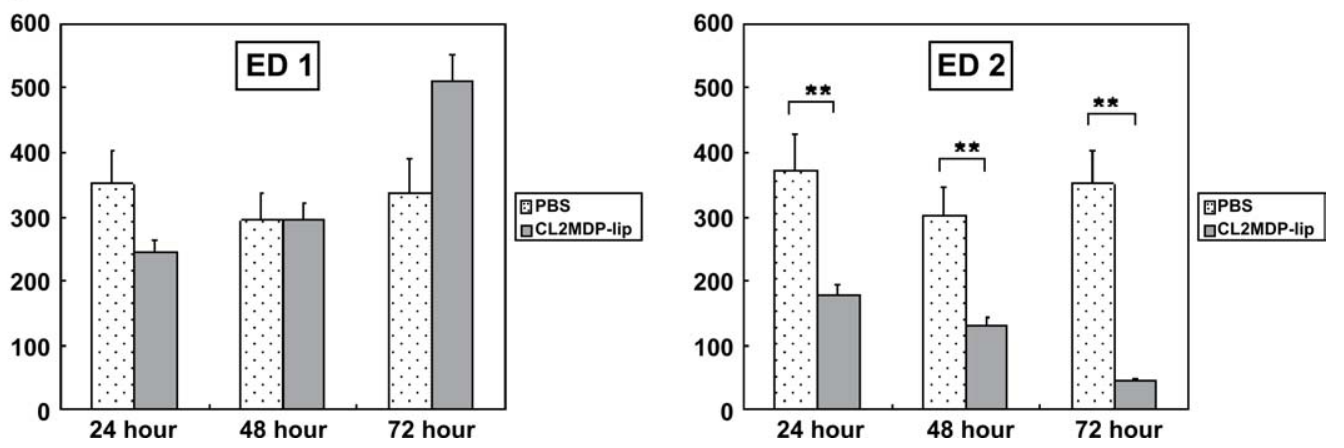

Fig. 1. Subconjunctival injection of $\mathrm{CL}_{2} \mathrm{MDP}$-lip decreased conjunctival macrophages but not splenic macrophages in naive $\mathrm{BN}$ rats. Naive $\mathrm{BN}$ rats were subconjunctivally injected with $\mathrm{CL}_{2}$ MDP-lip. 24, 48 or $72 \mathrm{~h}$ after injection, conjunctivas were harvested for detection of ED1- and ED2-positive cells by immunohistochemistry. (A) Photographs of sections of conjunctiva. Injection of $\mathrm{CL}_{2} \mathrm{MDP}$-lip decreased ED2- but not ED1-positive cells in the conjunctiva at all the time points. $\times 240$. (B) Cell counting of conjunctival macrophages. The number of ED2-positive cells was significantly decreased by subconjunctival injection of $\mathrm{CL}_{2} \mathrm{MDP}-l i p .{ }^{*} p<0.01$. (C) Photographs of sections of spleen. Subconjunctival injection of $\mathrm{CL}_{2} \mathrm{MDP}^{*}$-lip did not affect splenic macrophages. $\times 240$. (D) Cell counting of splenic macrophages. The number of splenic macrophages did not differ significantly between the groups.

( $2 \mathrm{ml}$ of each) was performed at the time of Ag challenge ( $n=10$ per group).

\subsection{Immunohistochemistry}

Sections for immunohistochemistry were prepared according to Kawamoto's method (Kawamoto and Shimizu, 2000). Briefly, the eyes were immediately frozen in hexane cooled with dry-ice and immersed in $3 \%$ carboxymethyl cellulose (CMC) gel, and then placed in cooled hexane again until the CMC gel was completely frozen. After trimming to the central horizontal section including the optic nerve, the surface of the CMC block was covered with a polyvinylidene chloride film (Asahikasei Kogyo, Tokyo, Japan) pre-coated with synthetic rubber cement (Cryoglue type 1, Finetec Co., LTD, Tokyo, Japan). Sections $4-\mu \mathrm{m}$ thick were cut with a cryomicrotome. The films with the sections were mounted on cooled slide glasses with double-sided adhesive tape (Nitoms, Inc., Tokyo, Japan). All samples were fixed for $5 \mathrm{~min}$ in cooled methanol, and then washed with $0.01 \mathrm{M}$ PBS, pH 7.4. Endogenous peroxidase activity was inhibited with $0.1 \% \mathrm{NaN}_{3}$ and $0.3 \% \mathrm{H}_{2} \mathrm{O}_{2}$ in distilled water for $10 \mathrm{~min}$ at room temperature. Samples were first exposed to Abs for $30 \mathrm{~min}$ and then to biotinylated RAMG for $1 \mathrm{~h}$. All slides were revealed using an Avidin-Biotin-Compex kit (Vector laboratories Inc., USA), and then developed with 3,3'-diaminobenzidine tetrahydrochloride (Sigma, St. Louis, MO). 
C

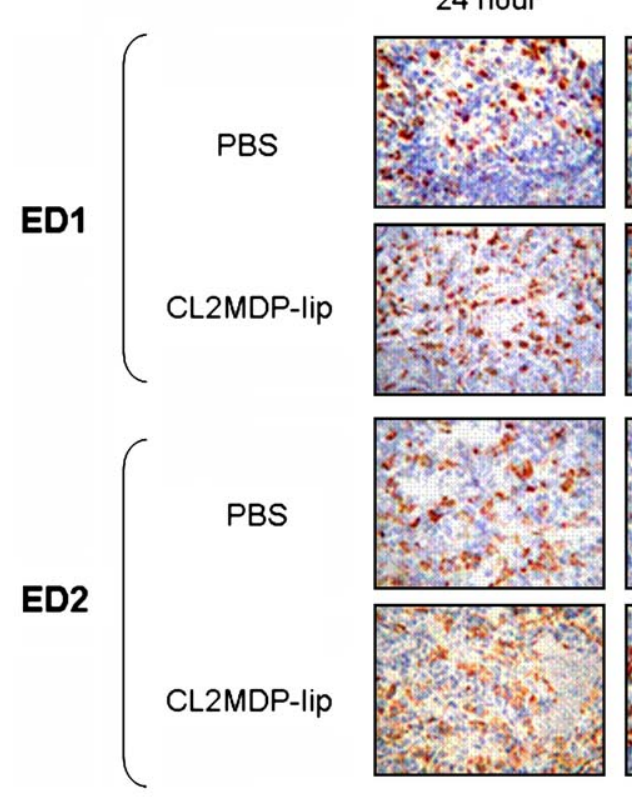

48 hour
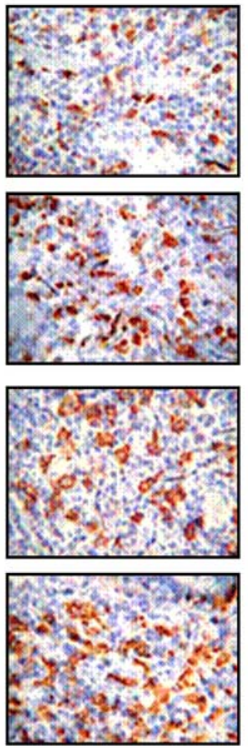

72 hour
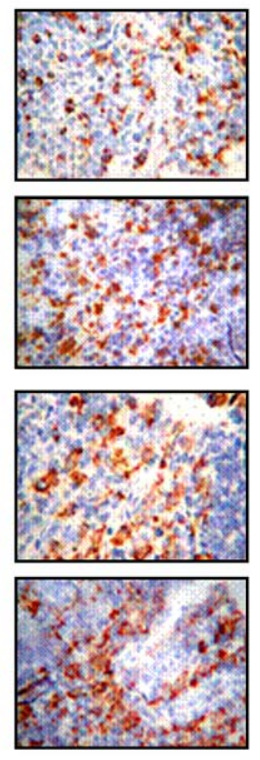

\section{D}
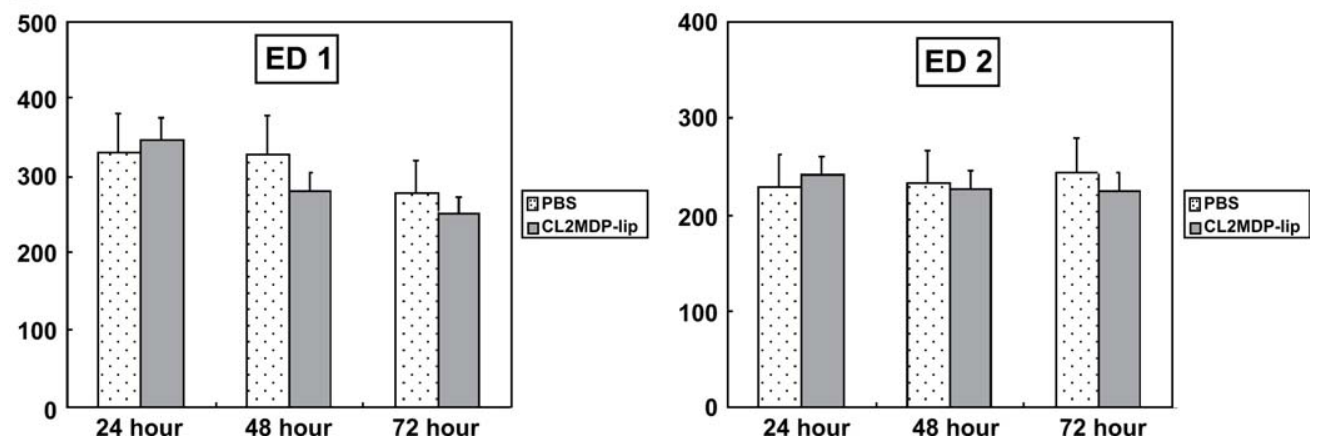

Fig. 1 (continued)

\subsection{Cell count and statistical analysis}

Infiltrating cells in the conjunctiva were counted throughout sections. ED1- and ED2-positive cells in the periarteriolar lymphoid sheath close to the germinal center of the spleen were counted per field. Three different sections per sample were counted. The data are presented as averages of different samples. Statistical comparisons of the number of Ab-stained cells were performed with the Mann-Whitney $U$-test.

\section{Results}

\subsection{Subconjunctival injection of $C L_{2} M D P$-lip decreases ED2-positive cells in the conjunctiva of naive $B N$ rats}

First, to examine the effects of $\mathrm{CL}_{2} \mathrm{MDP}$-lip on macrophages in the conjunctiva, we injected $\mathrm{CL}_{2} \mathrm{MDP}$ lip subconjunctivally into naive $\mathrm{BN}$ rats and harvested eyes at different time points after injection. Up to $72 \mathrm{~h}$ after injection, the number of ED2-positive cells was significantly less in the conjunctiva of rats treated with $\mathrm{CL}_{2}$ MDP-lip compared to the control group (Fig. 1A,B). In contrast, the number of ED1-positive cells was similar in the conjunctiva of both groups (Fig. 1A,B). To examine whether subconjunctival injection of $\mathrm{CL}_{2} \mathrm{MDP}$-lip induces local or systemic effects, macrophage numbers in spleens were evaluated by immunohistochemistry. The number of macrophages in spleens did not differ in any groups at any of the time points (Fig. 1C,D). Thus, subconjunctival injection of $\mathrm{CL}_{2}$ MDP-lip selectively decreases the number of conjunctival macrophages, especially ED2-positive cells.

\subsection{ED1- but not ED2-positive cells are increased in the conjunctiva by EC induction}

To examine the phenotypes of infiltrating macrophages, we induced EC by transfer of OVA-specific T cells. EC induction increased the numbers of ED1- but 
not ED2-positive cells compared to the number in naive rats (Fig. 2A). The EC-induced increase in ED1-positive cells was statistically significant, while the number of ED2-positive cells was not affected (Fig. 2B).

\subsection{Subconjunctival injection of $\mathrm{CL}_{2} \mathrm{MDP}$-lip decreases ED2-positive cells in the conjunctiva of rats developing $E C$}

To investigate whether $\mathrm{CL}_{2}$ MDP-lip decreases the number of macrophages in the conjunctiva of rats with EC, $\mathrm{CL}_{2}$ MDP-lip was subconjunctivally injected into EC-developing rats at different time points prior to Ag challenge. $\mathrm{CL}_{2} \mathrm{MDP}-$ lip significantly decreased the number of ED2-positive cells in the conjunctiva compared to the control group at all time points (Fig. 3B,E). However, the number of infiltrating ED1positive cells was inhibited only when $\mathrm{CL}_{2}$ MDP-lip was subconjunctivally injected just prior to $\mathrm{Ag}$ challenge (Fig. 3A,E). Similarly, the reduction in OX-6-positive cells was observed only when the treatment was
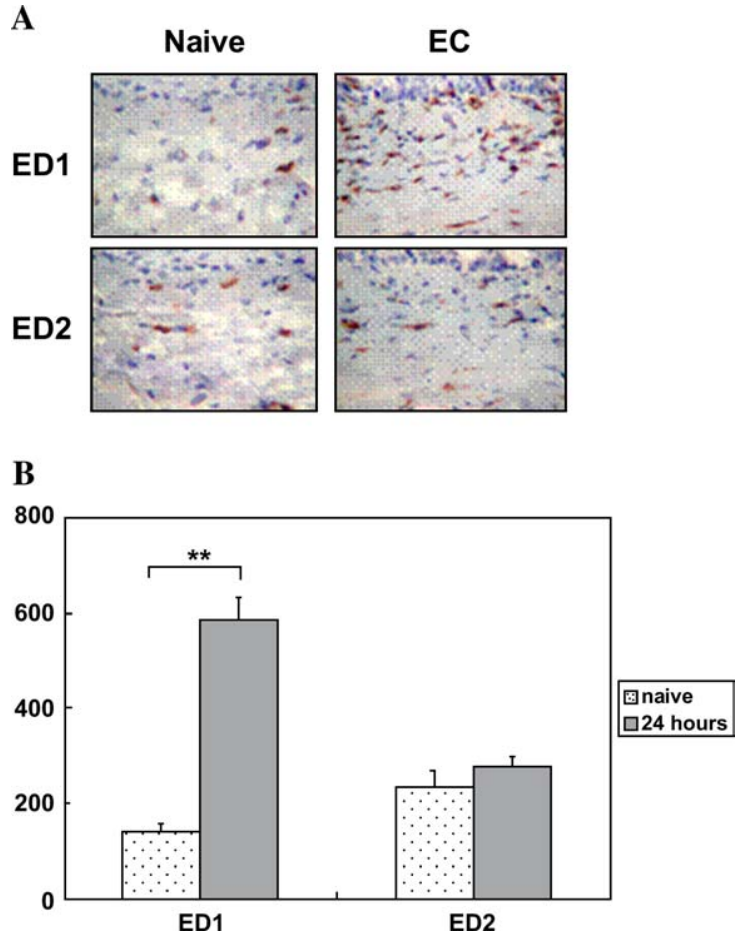

Fig. 2. The number of ED1- but not ED2-positive cells increased in the conjunctiva after induction of EC. OVA-specific $\mathrm{T}$ cells were transferred into $\mathrm{BN}$ rats. Four days later, the rats were challenged with OVA. Conjunctivas were harvested for immunohistochemical analysis $24 \mathrm{~h}$ after OVA challenge and compared to conjunctivas from naive rats. (A) Photographs of ED1- and ED2-positive cells in the conjunctiva. $\times 240$. The number of ED1-positive cells was increased by the induction of EC. The number of ED2-positive cells was similar between the two groups. (B) Cell counting of ED1- and ED2-positive cells in the conjunctiva. EC induction significantly increased the number of ED1-positive cells but did not change the number of ED2positive cells. ${ }^{* *} p<0.01$ performed just prior to Ag challenge (Fig. 3C,E). The number of MBP-positive cells in the conjunctiva was increased when the treatment was performed $24 \mathrm{~h}$ before or just prior to OVA challenge (Fig. 3D,E). Effects of $\mathrm{CL}_{2}$ MDP-lip on mast cells were little, if any (data not shown). Infiltration of inflammatory cells was greater in rats injected just prior to OVA challenge (Fig. 3A-E), compared to injections performed 24 or $48 \mathrm{~h}$ prior to OVA challenge. We believe the high permeability of OVA in the conjunctival substantial tissues accounts for this observation.

\subsection{Systemic $C L_{2} M D P$-lip treatment decreases the number of infiltrating macrophages in the conjunctiva}

Finally, we examined the effects of systemic $\mathrm{CL}_{2}$ MDP-lip treatment on macrophage infiltration into the conjunctiva. Intravenous injection of $\mathrm{CL}_{2} \mathrm{MDP}$-lip at the time of OVA challenge decreased the number of ED2-positive cells compared with the control (Fig. 4A,B). The numbers of ED1-positive and OX6positive cells were lower, but not significantly, than in the control group (Fig. 4A,B). The number of MBPpositive cells was not affected by treatment (Fig. 4A,B). The number of ED1- and ED2-positive cells in the spleen was decreased by $\mathrm{CL}_{2} \mathrm{MDP}$-lip treatment (Fig. 4C,D).

\section{Discussion}

Mast cells and eosinophils are major effector cells in AC (Church and McGill, 2002), but infiltration of macrophages also occurs in the conjunctiva, especially in severe types of AC such as AKC (Foster et al., 1991; Leonardi et al., 2000). The role of macrophages in the development of AC has not yet been extensively investigated. In our previous experiments using EC as a model for AKC (Fukushima et al., 2003), we demonstrated that macrophages infiltrate into the conjunctiva and residential macrophages express MHC class II molecules (submitted for publication). Therefore, we speculate that macrophages might be important in the development of EC. In this study, we investigated first whether conjunctival macrophages are functional APC by depleting residential macrophages by injecting $\mathrm{CL}_{2}$ MDP-lip subconjunctivally 24 and $48 \mathrm{~h}$ prior to OVA challenge. Second, to determine if $\mathrm{CL}_{2} \mathrm{MDP}$-lip inhibits infiltration of macrophages into the conjunctiva, we injected $\mathrm{CL}_{2}$ MDP-lip subconjunctivally or intravenously just prior to OVA challenge.

The inhibitory effect of $\mathrm{CL}_{2}$ MDP-lip on ED2positive cells (ED1-positive cells were unaffected) persisted in naive rats for at least $72 \mathrm{~h}$ in the conjunctiva 
A
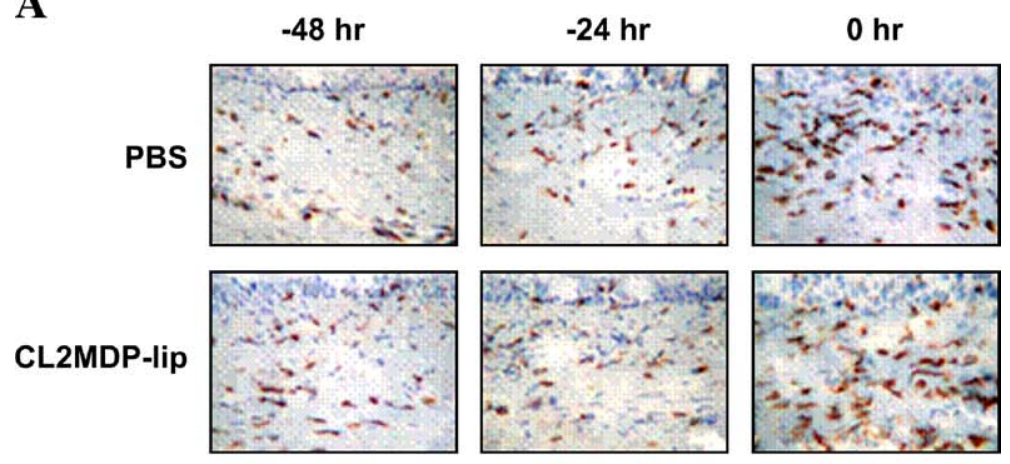

B
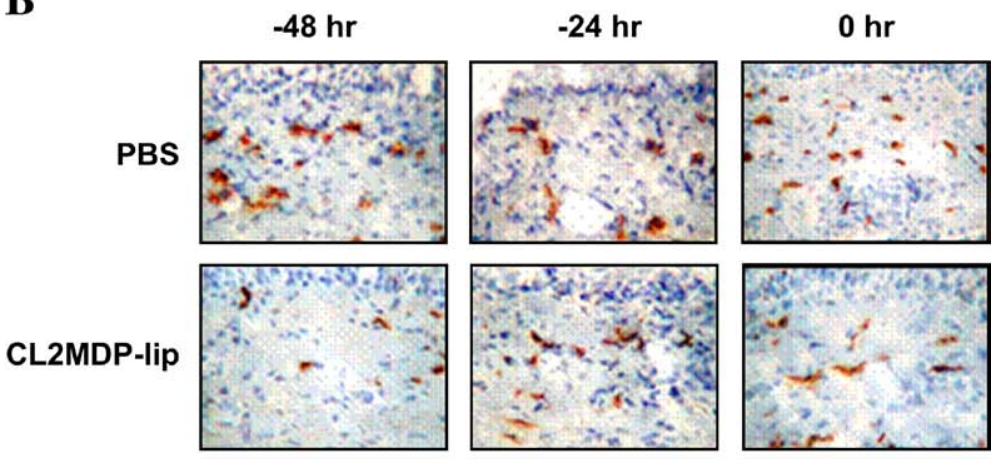

C
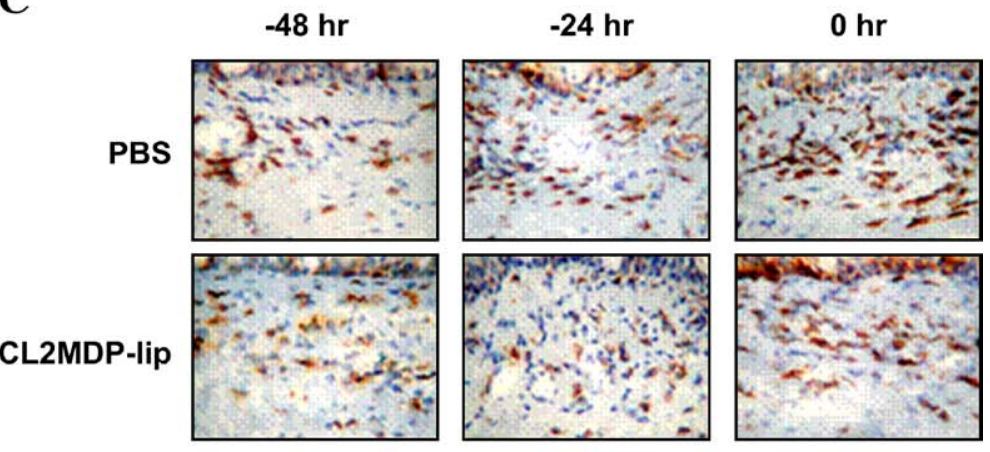

D
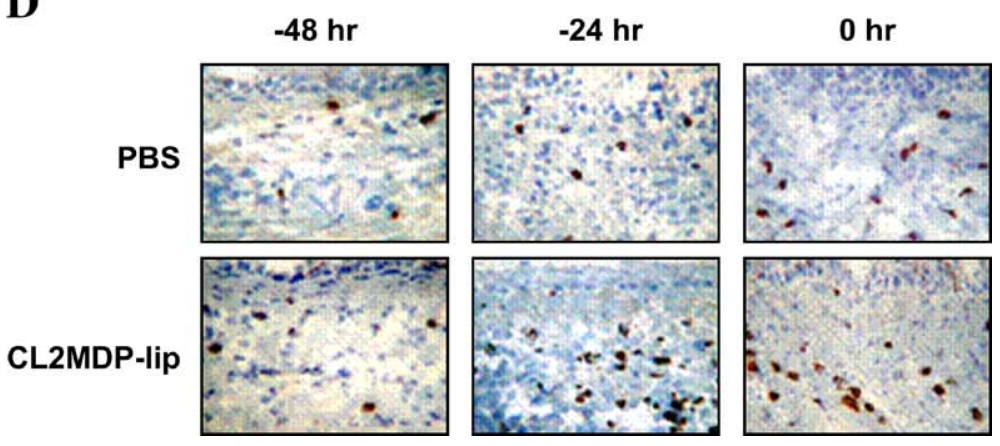

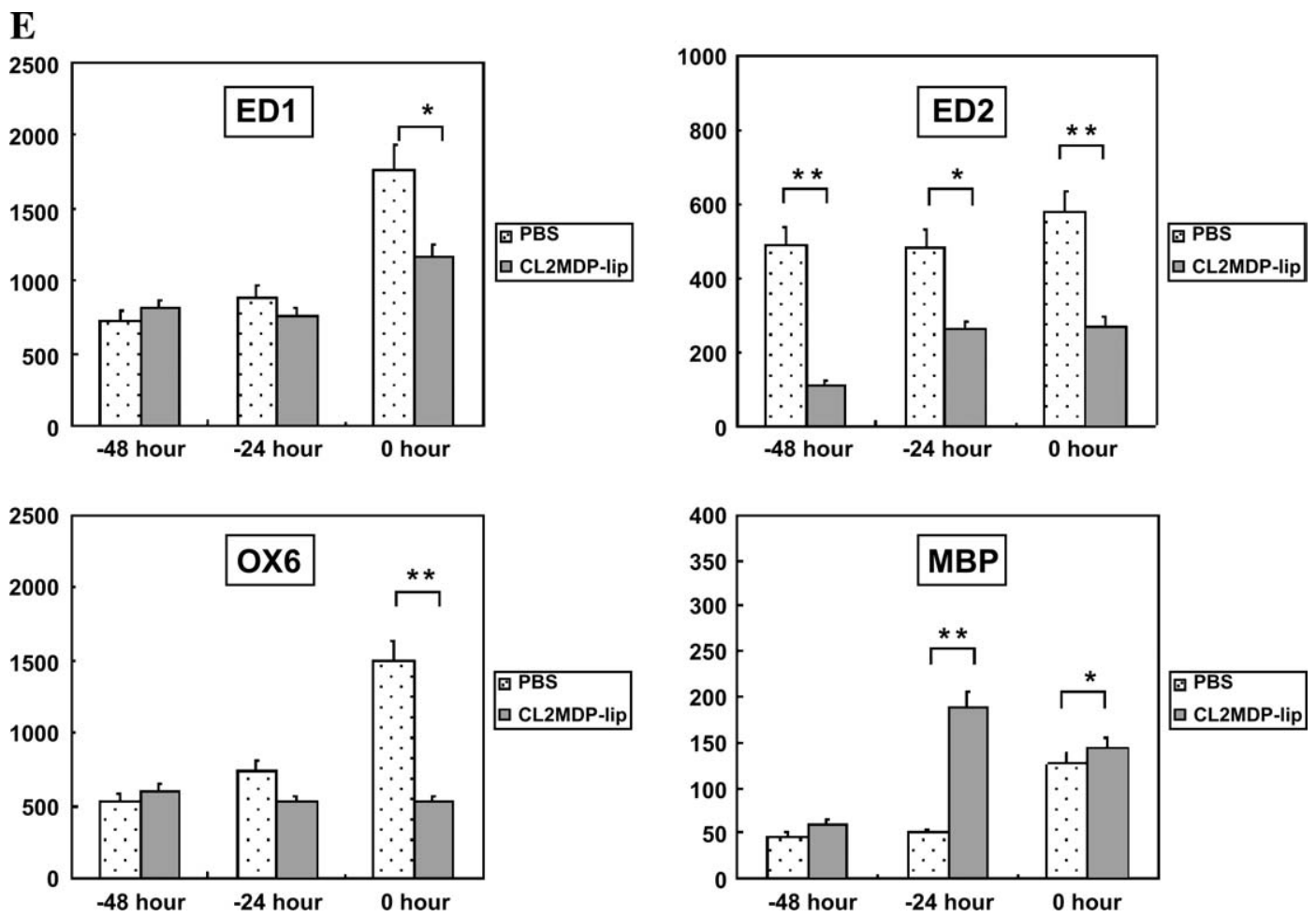

Fig. 3 (continued)

(Fig. 1). Treatment of EC-developing rats with $\mathrm{CL}_{2}$ MDP-lip at 24 or $48 \mathrm{~h}$ prior to $\mathrm{Ag}$ challenge did not decrease the number of ED1-positive cells, but decreased the number of ED2-positive cells in the conjunctiva (Fig. 3). These data suggest that ED2positive cells might not serve as APC in the conjunctiva, although residual ED2-positive cells not depleted by $\mathrm{CL}_{2}$ MDP-lip might have induced an allergic response. It is still unknown whether ED1-positive cells are APC.

ED1-positive cells are reportedly the infiltrating macrophages, while ED2-positive cells appear to be the residential macrophages (Dijkstra et al., 1985; van Rees et al., 1985). In addition, the precursors which do not phagocytize (directly after leaving the bone marrow) or phagocytize little (shortly before differentiation into mature macrophages) are positive for ED1, but not ED2 (Angelov et al., 1996). Our data demonstrating that numbers of ED1- but not ED2-positive cells in the conjunctiva are increased by the induction of EC (Fig. 2) suggest that ED1-positive cells infiltrated the conjunctiva, and ED2-positive cells are residential macrophages in the conjunctiva. Effects of $\mathrm{CL}_{2} \mathrm{MDP}$-lip on ED2positive cells in the conjunctiva were noted up to $72 \mathrm{~h}$ after injection (Fig. 1), even when EC was induced (Fig. 3). In contrast, the number of ED1-positive cells decreased only when the treatment was performed just prior to OVA challenge. The reason why $\mathrm{CL}_{2} \mathrm{MDP}$-lip treatment just prior to OVA challenge inhibited the infiltration of ED1-positive cells is still unclear. One possible explanation is that effects of $\mathrm{CL}_{2} \mathrm{MDP}$-lip in the conjunctiva persist for less than $24 \mathrm{~h}$ and, therefore, its effective timeframe is restricted. In any case, $\mathrm{CL}_{2} \mathrm{MDP}$ lip could be useful therapeutically for inhibiting infiltrating macrophages within $24 \mathrm{~h}$ of antigen challenge.

Fig. 3. Subconjunctival injection of $\mathrm{CL}_{2}$ MDP-lip decreased the number of conjunctival macrophages in EC-developing BN rats. OVA-specific T cells were transferred into BN rats. Four days later, the rats were challenged with OVA. Conjunctivas were harvested for immunohistochemical analysis $24 \mathrm{~h}$ after OVA challenge. $\mathrm{CL}_{2}$ MDP-lip was subconjunctivally injected 24 or $48 \mathrm{~h}$ before or just prior to OVA challenge. (A) Photographs of ED1positive cells in the conjunctiva. No apparent difference was noted between $\mathrm{CL}_{2} \mathrm{MDP}$-lip and PBS treated groups, although the number of ED1positive cells was slightly decreased in the $\mathrm{CL}_{2} \mathrm{MDP}$-lip treated group at $0 \mathrm{~h}$. $\times 240$. (B) Photographs of ED2-positive cells in the conjunctiva. $\mathrm{CL}_{2}$ MDP-lip treatment decreased the number of ED2-positive cells at all the treatment time points. $\times 240$. (C) Photographs of OX6-positive cells in the conjunctiva. Similar to the response of ED1-positive cells, $\mathrm{CL}_{2} \mathrm{MDP}$-lip slightly decreased the number of OX6-positive cells only at 0 h. $\times 240$. (D) Photographs of MBP-positive cells in the conjunctiva. The increase in MBP-positive cells induced by $\mathrm{CL}_{2} \mathrm{MDP}_{\text {-lip }}$ treatment was noted at -24 and 0 h. $\times 240$. (E) Cell counting of ED1-, ED2-, OX6- and MBP-positive cells. Cell counting confirmed the significant differences indicated as asterisks. ${ }^{*} p<0.05, * * p<0.01$. 
A
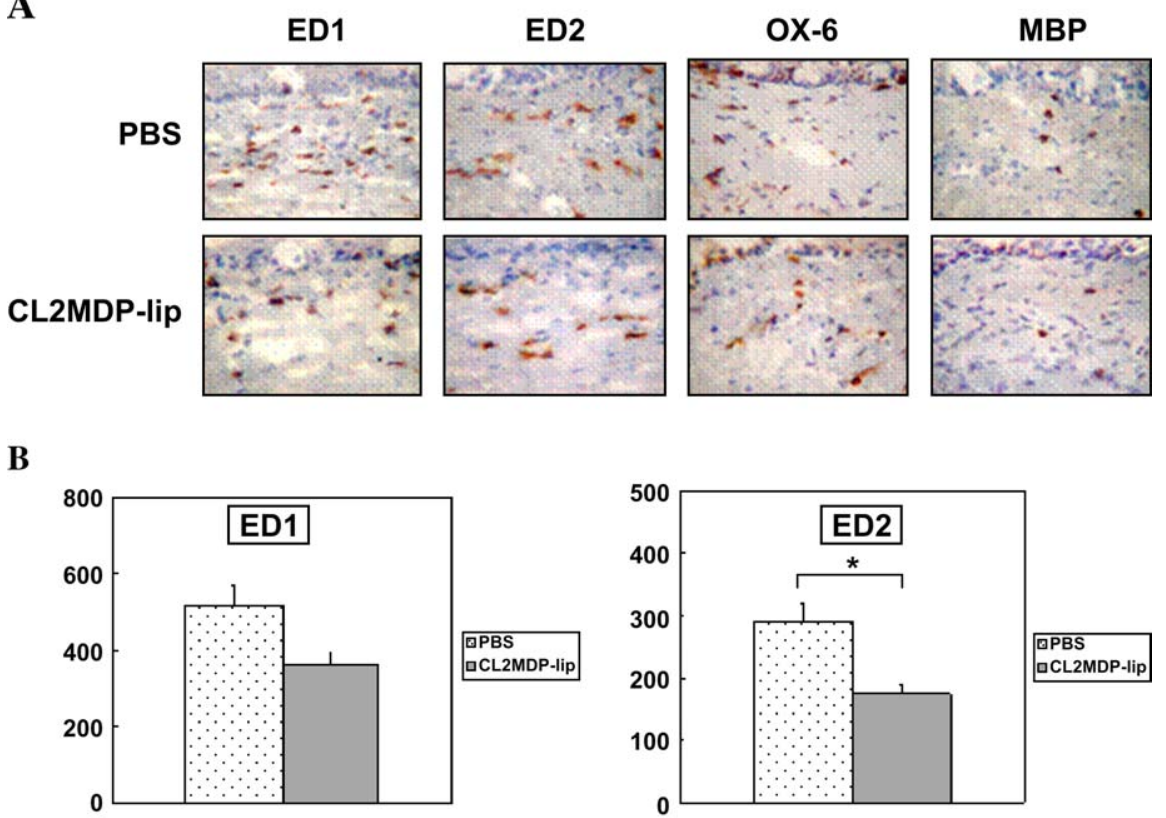

B
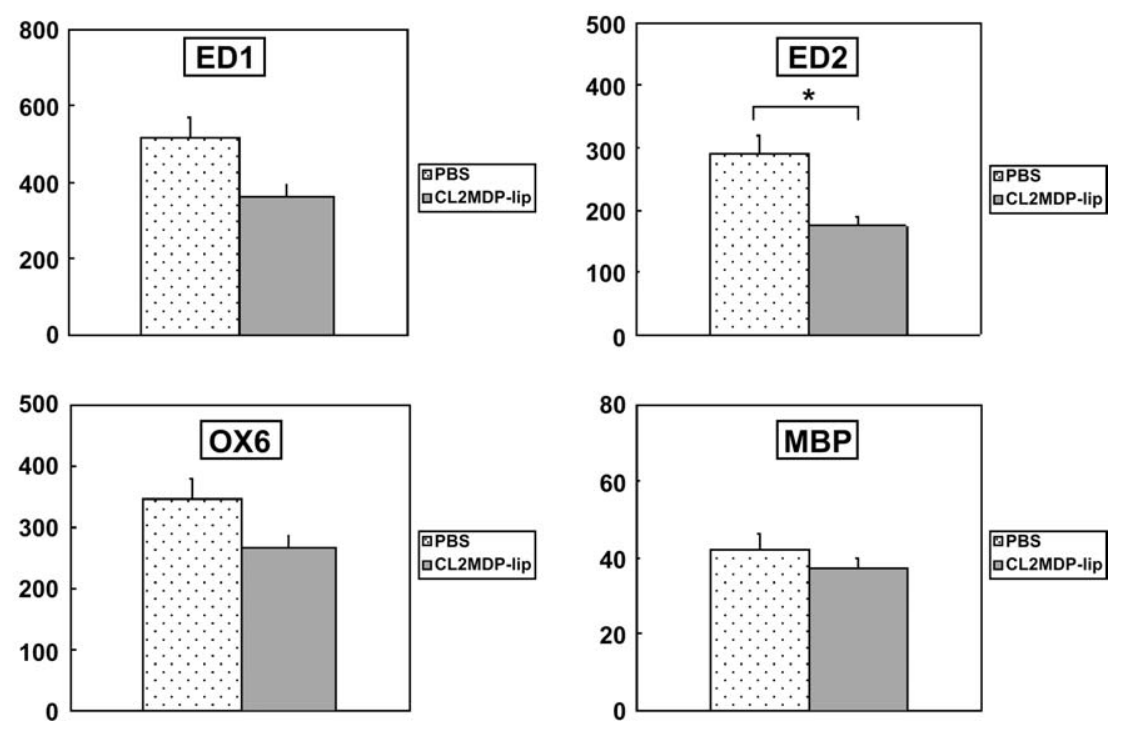

Fig. 4. Intravenous injection of $\mathrm{CL}_{2} \mathrm{MDP}$-lip decreased the number of ED2-positive conjunctival macrophages in EC-developing BN rats. OVAspecific $\mathrm{T}$ cells were transferred into $\mathrm{BN}$ rats. Four days later, the rats were challenged with OVA. Conjunctivas were harvested for immunohistochemical analysis $24 \mathrm{~h}$ after OVA challenge. $\mathrm{CL}_{2}$ MDP-lip was injected intravenously at the time of OVA challenge. (A) Photographs of ED1-, ED2-, OX6- and MBP-positive cells in the conjunctiva. Intravenous injection of $\mathrm{CL}_{2} \mathrm{MDP}$-lip decreased the number of ED2-positive cells in the conjunctiva, whereas numbers of ED1-, OX6- or MBP-positive cells were not affected. $\times 240$. (B) Cell counting of ED1-, ED2-, OX6- and MBPpositive cells in the conjunctiva. Cell counting confirmed that only the number of ED2-positive cells differed significantly. ${ }^{*} p<0.05$. (C) Photographs of ED1- and ED2-positive cells in the spleen. Numbers of both ED1- and ED2-positive cells were decreased by $\mathrm{CL}_{2} \mathrm{MDP}$-lip treatment. $\times 240$. (D) Cell counting of ED1- and ED2-positive cells in the spleen. Cell counting confirmed both ED1- and ED2-positive cells were significantly decreased by $\mathrm{CL}_{2}$ MDP-lip treatment. ${ }^{* *} p<0.01$.

Although the effects of $\mathrm{CL}_{2} \mathrm{MDP}-$ lip on conjunctival macrophages have been partially elucidated, the regulating mechanisms of macrophage accumulation in the conjunctiva have not been examined. Several studies have demonstrated that molecules such as macrophagecolony-stimulating factor (Razzaque et al., 2002) and MIF (Razzaque et al., 2004) are involved in regulation of macrophage infiltration into the conjunctiva of OCP. Studying the expression of these molecules in the conjunctiva of EC is important to better understand how $\mathrm{CL}_{2}$ MDP-lip affects the infiltration of conjunctival macrophages. ED2-positive residential macrophages may also produce inflammatory molecules and, therefore, inhibition of ED2-positive cells by $\mathrm{CL}_{2} \mathrm{MDP}$-lip might be of value in suppressing inflammatory processes in the conjunctiva.
Systemic (intravenous administration) treatment with $\mathrm{CL}_{2}$ MDP-lip inhibited both ED1- and ED2-positive cells in the spleen, although not completely. Similarly, infiltration of both ED1- and ED2-positive cells into the conjunctiva was suppressed, but only the number of ED2-positive cells was significantly decreased. Thus, infiltrating macrophages in the conjunctiva originated from systemic circulating monocytes or macrophages.

An interesting but unresolved issue is that subconjunctival injection of $\mathrm{CL}_{2} \mathrm{MDP}$-lip increased the number of eosinophils in EC. This increase may have been due to the Th1/Th2 balance in the conjunctiva (Fukushima et al., 2003); macrophages produce IFN- $\gamma$ and the lower relative concentration of IFN- $\gamma$ due to the smaller number of macrophages following treatment with $\mathrm{CL}_{2}$ MDP-lip might have enhanced Th2 cytokines 
C

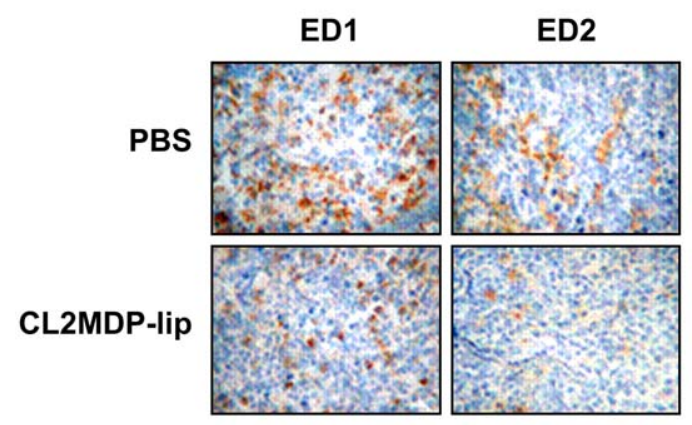

D
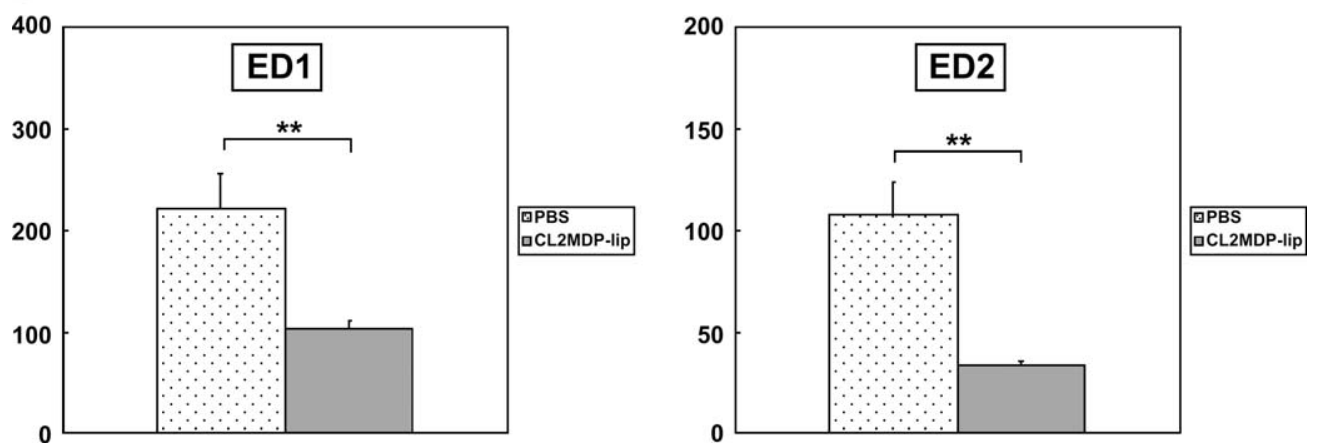

Fig. 4 (continued)

(Gajewski et al., 1989; Sekine et al., 1997). Clarification is imperative because eosinophils are major effector cells in the development of AC.

In conclusion, $\mathrm{CL}_{2} \mathrm{MDP}-\mathrm{lip}$ suppresses infiltration of macrophages into the conjunctiva during the development of EC and may become a useful therapeutic tool in managing macrophage-mediated conjunctival diseases such as AKC.

\section{References}

Angelov DN, Neiss WF, Streppel M, Walther M, Guntinas-Lichius O, Stennert E. ED2-positive perivascular cells act as neuronophages during delayed neuronal loss in the facial nucleus of the rat. Glia 1996;16:129-39.

Broekhuyse RM, Huitinga I, Kuhlmann ED, Rooijen NV, Winkens HJ. Differential effect of macrophage depletion on two forms of experimental uveitis evoked by pigment epithelial membrane protein (EAPU), and by melanin-protein (EMIU). Exp Eye Res 1997;65:841-8.

Church MK, McGill JI. Human ocular mast cells. Curr Opin Allergy Clin Immunol 2002;2:419-22.

Dijkstra CD, Dopp EA, Joling P, Kraal G. The heterogeneity of mononuclear phagocytes in lymphoid organs: distinct macrophage subpopulations in the rat recognized by monoclonal antibodies ED1, ED2 and ED3. Immunology 1985;54:589-99.

El-Asrar AM, Struyf S, Al-Kharashi SA, Missotten L, Van Damme J, Geboes K. Expression of $\mathrm{T}$ lymphocyte chemoattractants and activation markers in vernal keratoconjunctivitis. Br J Ophthalmol 2002;86:1175-80

Foster CS, Sainz De La Maza M. Ocular cicatricial pemphigoid review. Curr Opin Allergy Clin Immunol 2004;4:435-9.
Foster CS, Rice BA, Dutt JE. Immunopathology of atopic keratoconjunctivitis. Ophthalmology 1991;98:1190-6.

Fukushima A, Ozaki A, Fukata K, Ishida W, Ueno H. Ag-specific recognition, activation, and effector function of $\mathrm{T}$ cells in the conjunctiva with experimental immune-mediated blepharoconjunctivitis. Invest Ophthalmol Vis Sci 2003;44:4366-74.

Gajewski TF, Schell SR, Nau G, Fitch FW. Regulation of T-cell activation: differences among T-cell subsets. Immunol Rev 1989; 111:79-110.

Iwamoto H, Nishino K, Magone TM, Whitcup SM, Yoshida O, Yoshida $\mathrm{H}$, et al. Experimental immune-mediated blepharoconjunctivitis in rats induced by immunization with ragweed pollen. Graefes Arch Clin Exp Ophthalmol 2000;238:346-51.

Kawamoto T, Shimizu M. A method for preparing 2- to 50-micronthick fresh-frozen sections of large samples and undecalcified hard tissues. Histochem Cell Biol 2000;113:331-9.

Leonardi A, Brun P, Tavolato M, Abatangelo G, Plebani M, Secchi AG. Growth factors and collagen distribution in vernal keratoconjunctivitis. Invest Ophthalmol Vis Sci 2000;41: 4175-81.

Metz DP, Hingorani M, Calder VL, Buckley RJ, Lightman SL. T-cell cytokines in chronic allergic eye disease. J Allergy Clin Immunol 1997; 100:817-24.

Polfliet MM, van de Veerdonk F, Dopp EA, van KesterenHendrikx EM, van Rooijen N, Dijkstra CD, et al. The role of perivascular and meningeal macrophages in experimental allergic encephalomyelitis. J Neuroimmunol 2002; 122:1-8.

Razzaque MS, Foster CS, Ahmed AR. Role of enhanced expression of $\mathrm{m}$-CSF in conjunctiva affected by cicatricial pemphigoid. Invest Ophthalmol Vis Sci 2002;43:2977-83.

Razzaque MS, Foster CS, Ahmed AR. Role of macrophage migration inhibitory factor in conjunctival pathology in ocular cicatricial pemphigoid. Invest Ophthalmol Vis Sci 2004; 45:1174-81. 
van Rees EP, Dopp EA, Dijkstra CD, Sminia T. The postnatal development of cell populations in the rat popliteal lymph node. An immunohistochemical study. Cell Tissue Res 1985; 242:391-8.

Sakurai E, Anand A, Ambati BK, van Rooijen N, Ambati J. Macrophage depletion inhibits experimental choroidal neovascularization. Invest Ophthalmol Vis Sci 2003;44:3578-85.

Sekine Y, Bowen LK, Heidler KM, Van Rooijen N, Brown JW, Cummings OW, et al. Role of passenger leukocytes in allograft rejection: effect of depletion of donor alveolar macrophages on the local production of TNF-alpha, T helper 1/T helper 2 cytokines, $\mathrm{IgG}$ subclasses, and pathology in a rat model of lung transplantation. J Immunol 1997;159:4084-93.

Van Rooijen N, Sanders A. Liposome mediated depletion of macrophages: mechanism of action, preparation of liposomes and applications. J Immunol Methods 1994;174:83-93.

Yoshida O, Yoshida H, Iwamoto H, Nishino K, Fukushima A, Ueno H. Genetic background determines the nature of immune responses and experimental immune-mediated blepharoconjunctivitis (EC). Curr Eye Res 1999;18:117-24. 